\title{
К теории плазмон-экситонов: оценка константы взаимодействия и оптический спектр
}

\author{
(C) Н.С. Аверкиев, А.В. Коротченков , В.А. Кособукин \\ Физико-технический институт им. А.Ф. Иофрфе Российской академии наук, \\ 194021 Санкт-Петербург, Россия \\ `E-mail: KorotchenkovAlex@gmail.com
}

Поступила в Редакцию 18 марта 2019 г.

В окончательной редакции 28 марта 2019 г.

Принята к публикации 28 марта 2019 г.

\begin{abstract}
Теоретически изучалась структура оптического спектра, связанная с резонансным взаимодействием квазидвумерных экситонов и локальных плазмонов. Константа плазмон-экситонного взаимодействия оценена в модели близко расположенных квантовой ямы в полупроводнике и слоя металлических наночастиц в соседнем диэлектрике. Численным расчетом для наносистем $\mathrm{GaAs} / \mathrm{Ag}$ и $\mathrm{ZnO} / \mathrm{Al}$ показано, что при плазмонэкситонном резонансе спектр имеет двухпиковую структуру, поведение которой при расстройке резонанса соответствует антипересечению спектров плазмонов и экситонов, имеющему признаки расщепления Раби.
\end{abstract}

Ключевые слова: плазмоны, экситоны, смешанные возбуждения, наноструктуры, антипересечение спектров.

DOI: $10.21883 / F T P .2019 .08 .47996 .9107$

Ранее было установлено, что смешанные плазмонэкситонные возбуждения образуются при резонансном взаимодействии локальных плазмонов с молекулярными экситонами Френкеля или низкоразмерными экситонами Ванье. В ряде работ (например, [1,2] и ссылки в них) наблюдалось плазмон-экситонное расщепление Раби большой величины в оптических спектрах композитов, образованных молекулярными $J$-агрегатами и металлическими наноструктурами. Для экситонов большого радиуса аналогичные эффекты исследовались сравнительно мало [3,4]. О наблюдении расщепления Раби сообщалось в работе [4], где исследовались оптические спектры квантовой ямы на основе $\mathrm{ZnO}$, находящейся вблизи слоя нанодисков Al. Теория плазмон-экситонов развивалась в работах $[5,6]$, посвященных спектроскопии упругого рассеяния света на металлической наночастице, находящейся вблизи квантовой ямы, и отражения света от слоя таких частиц. Развитие этого нового направления спектроскопии делает актуальной систематическую оценку плазмон-экситонного расщепления в оптических спектрах наноструктур металлполупроводник.

Цель данной работы - оценка константы взаимодействия локальных плазмонов с квазидвумерными (квази-2D) экситонами и анализ расщепления оптического спектра при резонансе этих возбуждений.

Рассматривается модель, показанная схематически на рис. 1. В среде с проницаемостью $\varepsilon_{1}$ на расстоянии $h_{1}$ от поверхности $z=0$ находится слой металлических наночастиц. Предполагается, что частицы в форме одинаковых эллипсоидов вращения (сфероидов) с длинами полуосей $a$ в плоскости $x y$ и $\eta a$ вдоль оси вращения $z$ заполняют решетку с периодом $A$ в плоскости $z=-h_{1}<0$. В соседнем полупроводнике с фоновой диэлектрической проницаемостью $\varepsilon_{2}=\varepsilon_{b}$ на расстоянии $h_{2}$ от его поверхности $z=0$ расположена квантовая яма с шириной $l$ и средней плоскостью $z=h_{2}>0$. Все геометрические параметры считаются малыми по сравнению с длиной волны света $\sim 1 / k_{0}$, где $k_{0}=\omega / c, \omega-$ частота, $c-$ скорость света. При этом в квазистатическом приближении электродинамики исследуются возбуждения, образованные дипольными плазмонами наночастиц из среды 1 и нерадиационными („темными“) экситонами квантовой ямы из среды 2. Связанные моды плазмон-экситонов образуются вследствие кулоновского взаимодействия возбуждений через границу $z=0$.

В отсутствие наночастиц структура с квантовой ямой (рис. 1) изотропна и трансляционно инвариантна по $\boldsymbol{\rho}=(x, y)$ в плоскости $x y$. Для нее решениями уравнений Максвелла являются волны $\sim \exp (i \boldsymbol{\kappa} \cdot \boldsymbol{\rho})$ с поляризацией $p$ или $s$, которые в случае $\boldsymbol{\kappa}=\kappa \mathbf{e}_{x}$ имеют компоненты $\mathbf{E}^{p}=\left(E_{x}, 0, E_{z}\right)$ и $\mathbf{E}^{s}=\left(0, E_{y}, 0\right)$ с проекциями $E_{\alpha}(z ; \kappa) \exp (i \kappa x)$.

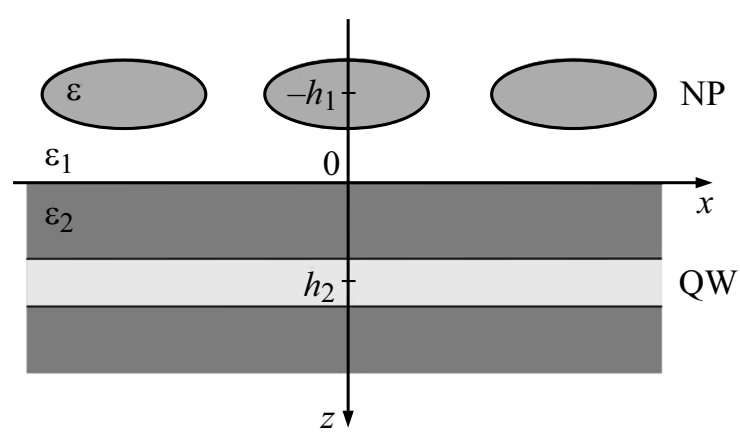

Рис. 1. Геометрия задачи: $\mathrm{QW}-$ квантовая яма, $\mathrm{NP}-$ слой наночастиц. 
Применительно к модели рис. 1 рассматриваем $p$-поляризованные волны, которые имеют нормальную к квантовой яме компоненту поля $E_{z}$, необходимую для образования при $\kappa \gg k_{0}$ кулоновских плазмон-экситонных мод. Вкладом $s$-поляризованного поля, который в квазистатическом приближении имеет малость $\sim k_{0}^{2} / \kappa^{2} \ll 1[5,6]$, далее пренебрегаем, а индекс поляризации $p$ в формулах опускаем. Методически мы сначала находим волны с $p$-поляризацией при $\kappa<\sqrt{\varepsilon_{n}} k_{0}$, $k_{n}=\sqrt{\varepsilon_{n} k_{0}^{2}-\kappa^{2}}, n=1,2$ (рис. 1) при использовании результатов теории квази-2D экситонов [7], а затем переходим к квазистатическому приближению для описания „темных“ экситонов с $\kappa \gg k_{0}, k_{n} \approx i \kappa$.

Компоненты поля, возбуждаемого в среде $1(z<0)$ без частиц, при $\kappa<\sqrt{\varepsilon_{1}} k_{0}$ равны

$$
E_{x}(z ; \kappa)=E_{x}^{\mathrm{inc}}(\kappa)\left(e^{i k_{1} z}+r e^{-i k_{1} z}\right)
$$

и $E_{z}=\left(i \kappa / k_{1}^{2}\right) d E_{x} / d z$. В формулу (1) входит коэффициент отражения света в среду 1

$$
r(\kappa)=\frac{r_{12}+r_{Q W} e^{2 i k_{2} h_{2}}}{1+r_{12} r_{Q W} e^{2 i k_{2} h_{2}}} .
$$

Он выражается через коэффициенты отражения света $r_{12}=\left(\varepsilon_{1} k_{2}-\varepsilon_{2} k_{1}\right) /\left(\varepsilon_{1} k_{2}+\varepsilon_{2} k_{1}\right)$ от поверхности $z=0$ полупроводника с $\varepsilon_{2}=\varepsilon_{b}, k_{2}=k_{b}$ и $r_{Q W}$ - от квантовой ямы в однородном полупроводнике. Для модели квантовой ямы с нелокальной поляризацией квази-2D экситонов в приближении эффективной массы, следуя [7], получаем

$$
r_{Q W}=\frac{i \Gamma_{0, x}}{\omega_{x}-\omega-i \Gamma-i \Gamma_{0, x}}, \quad \Gamma_{0, x}=\omega_{L T} k_{b} L
$$

В этом выражении сохранен только резонансный вклад c проекцией $x$, так как мы рассматриваем экситоны, имеющие изотропную дипольную поляризацию в плоскости квантовой ямы $x y$. В формуле (3) $\omega_{x}$ - частота квази-2D экситона с учетом размерного квантования электрона и дырки, их кулоновского взаимодействия и радиационного сдвига [7]. Далее, Г и $\Gamma_{0, x}-$ параметры нерадиационного и радиационного затухания квази-2D экситона, в последнем $\omega_{L T}-$ продольно-поперечное расщепление 3D-экситона с боровским радиусом $a_{\mathrm{B}}$ в материале квантовой ямы. Длина $L=\pi a_{\mathrm{B}}^{3} I_{c}^{2} / 2 \sim l$ определяется интегралом $I_{c}=\int d z \cos \left(k_{b} z\right) \Phi(z)$ от огибающей волновой функции $\Phi(z)=\Phi(-z)$ основного состояния квази-2D экситона, взятой при совпадающих координатах электрона и дырки в квантовой яме шириной $l$. Соотношение между и $L$ и $l$ служит мерой увеличения силы осциллятора квази-2D экситона (параметра радиационного затухания $\Gamma_{0, x}$ при $\left.\kappa=0\right)$ при уменьшении $l$ [7-9].

Для металлического наносфероида, находящегося в однородной среде с проницаемостью $\varepsilon_{1} \quad\left(\operatorname{Im} \varepsilon_{1}=0\right)$, компоненты диагонального тензора поляризуемости дипольного плазмона с поляризацией вдоль $\alpha$-полуоси сфероида, имеют вид [10]

$$
\chi_{\alpha \alpha}^{0}(\omega)=\frac{\eta a^{3}}{3} \frac{\left(U_{\alpha}^{0}\right)^{2}}{\left(u_{\alpha}^{0}\right)^{2}-\omega^{2}-i \omega \gamma} .
$$

Формула (4) получена для металла с диэлектрической функцией $\varepsilon(\omega)=\varepsilon_{\infty}-\omega_{p}^{2} /\left(\omega^{2}+i \omega \gamma\right)$ металла, где $\omega_{p}$ - плазменная частота, $1 / \gamma-$ время релаксации электронов, $\varepsilon_{\infty}-$ константа. Частоты $u_{\alpha}^{0}=$ $=\omega_{p} \sqrt{N^{(\alpha)} / \varepsilon_{*}^{(\alpha)}}$ и $U_{\alpha}^{0}=\omega_{p} \sqrt{\varepsilon_{1}} / \varepsilon_{*}^{(\alpha)}$ с $\varepsilon_{*}^{(\alpha)}=\left(\varepsilon_{\infty}-\varepsilon_{1}\right) N^{(\alpha)}$ $+\varepsilon_{1}$ зависят от отношения $\eta$ длин полуосей сфероида (его формы) через коэффициент деполяризации $N^{(\alpha)}$ $\left(0<N^{(\alpha)}<1,2 N^{(x)}+N^{(z)}=1\right)$. В соответствии с рис. 1 далее рассматривается монослой одинаковых сфероидов с центрами в узлах $\boldsymbol{\rho}_{\mathbf{n}}=A \mathbf{n}$ квадратной решетки с периодом $A$ в плоскости $z=-h_{1}<0$, где $\mathbf{n}=n_{x} \mathbf{e}_{x}+n_{y} \mathbf{e}_{y}$, $n_{\alpha}$ - целые числа.

Кулоновское взаимодействие дипольных плазмонов слоя наночастиц с квази-2D экситонами квантовой ямы (рис. 1) может приводить к образованию смешанных возбуждений. При их рассмотрении квазистатическое решение задачи (1)-(3) для экситонов является нулевым приближением, а слой точечных диполей с плазмонными поляризуемостями (4) считается возмущением. При учете этого резонансного возмущения в рамках самосогласованной теории $[5,6]$ приходим к представлению о плазмон-экситонных комплексах, связанных с узлами $\boldsymbol{\rho}_{\mathbf{n}}$ решетки. Такой комплекс образован дипольным плазмоном, который индуцируется на наночастице слоя в локальном (действующем) поле, включающем вклады полей других частиц слоя и их „изображений“, обусловленных поляризацией поверхности полупроводника и экситонной поляризацией квантовой ямы. При возбуждении $p$-поляризованным светом когерентная мода плазмон-экситонов в решетке $\boldsymbol{\rho}=\boldsymbol{\rho}_{\mathbf{n}}$ определяет длинноволновый оптический отклик в излучении наноструктур, схематически показанных на рис. 1.

Следуя результатам работ $[5,6]$, в приближении среднего поля $[10,11]$ после преобразования Фурье по $\boldsymbol{\kappa}$ в координатном представлении получаем квазистатическое уравнение вида $\chi=\chi^{0}+\chi^{0}(\Sigma U) \chi$. Здесь $\chi$ обозначает компоненты диагонального тензора дипольной поляризуемости плазмон-экситонного комплекса, а сумма $\Sigma U$ учитывает вклад индуцированных диполей в локальное (действующее) поле. Для рассматриваемой модели (рис. 1) находим компоненты $\chi_{\alpha \beta}=\delta_{\alpha \beta} \chi_{\alpha \alpha}$ с $\alpha=x$ или $y$ в виде

$$
\begin{aligned}
\chi_{\alpha \alpha}= & \left\{\frac{1}{\chi_{\alpha \alpha}^{0}}-\frac{1}{A^{3}}\left[S_{0}(0)+\mu S_{1}\left(\frac{2 h_{1}}{A}\right)\right]\right. \\
& \left.+\frac{6 h L}{A^{5}}\left(1-\mu^{2}\right) \frac{\omega_{L T}}{\omega_{\alpha}-\omega-i \Gamma} S_{2}\left(\frac{2 h}{A}\right)\right\}^{-1} .
\end{aligned}
$$

Здесь $\mu=\left(\varepsilon_{1}-\varepsilon_{b}\right) /\left(\varepsilon_{1}+\varepsilon_{b}\right)-$ коэффициент $r_{12}$ из формулы (2) в квазистатическом приближении $\left(\kappa \gg k_{0}\right)$ 
Параметры экситонов и плазмонов, использованные при вычислении $\chi_{\alpha \alpha}$ по формуле $(8)$ и $\Delta=W^{2} /\left(2 \omega_{0}\right)$ по формуле $(9)$

\begin{tabular}{|c|c|c|c|c|c|c|c|c|c|}
\hline Наноструктура & $\begin{array}{c}\hbar \omega_{0}, \\
\ni \mathrm{B}\end{array}$ & $\begin{array}{c}\hbar \omega_{L T}, \\
\text { мэB }\end{array}$ & $\begin{array}{l}a_{\mathrm{B}}, \\
\mathrm{HM}\end{array}$ & $\varepsilon_{b}$ & $\begin{array}{c}\hbar \Gamma, \\
\text { мэВ }\end{array}$ & $\begin{array}{l}a, \mathrm{HM} \\
(\eta)\end{array}$ & $\begin{array}{c}\hbar U_{\alpha} \\
\ni \mathrm{B}\end{array}$ & $\begin{array}{l}\hbar G_{\alpha}, \\
\mathrm{мэВ}\end{array}$ & $\begin{array}{l}\hbar \Delta, \\
\text { мэВ }\end{array}$ \\
\hline $\mathrm{GaAs} / \mathrm{Ag}$ & 1.51 & 0.08 & 14 & 12.5 & 0.3 & $\begin{array}{c}10 \\
(\eta=0.148)\end{array}$ & 5.92 & 30 & 2.7 \\
\hline $\mathrm{ZnO} / \mathrm{Al}$ & 3.4 & 2 & 1.8 & 6.7 & 3 & $\begin{array}{c}10 \\
(\eta=0.158)\end{array}$ & 13.6 & 520 & 12 \\
\hline
\end{tabular}

и $h=h_{1}+h_{2}$. Суммы

$$
\begin{gathered}
S_{0}(\xi)=\sum_{\mathbf{n}(\neq 0)} \frac{3 n_{\alpha}^{2}-\mathbf{n}^{2}-\xi^{2}}{\left(\mathbf{n}^{2}+\zeta^{2}\right)^{5 / 2}}, S_{1}(\xi)=-\frac{1}{\xi^{3}}+S_{0}(\xi), \\
S_{2}(\xi)=-\frac{1}{\xi^{5}}+\sum_{\mathbf{n}(\neq 0)} \frac{5 n_{\alpha}^{2}-\mathbf{n}^{2}-\xi^{2}}{\left(\mathbf{n}^{2}+\xi^{2}\right)^{7 / 2}}
\end{gathered}
$$

при $\alpha=x, y$ берутся по узлам $\boldsymbol{\rho}_{\mathbf{n}}=A \mathbf{n}$ решетки с $\mathbf{n}^{2}=n_{x}^{2}+n_{y}^{2}$. Они выражают вклады в локальное поле, действующее на выделенный $\alpha$-поляризованный плазмонный диполь, выбранный в точке $\rho_{\mathbf{n}=0}=0, z=-h_{1}$. Сумма $S_{0}(0) \approx 4.51$ относится к диполям слоя наночастиц с центрами $\boldsymbol{\rho}=\boldsymbol{\rho}_{\mathbf{n} \neq 0}$, а суммы $S_{1}<0$ при $\xi=2 h_{1} / A$ и $S_{2}<0$ при $\xi=2 h / A$ учитывают вклады зарядов „изображения“, обусловленных динамической поляризацией границы раздела $z=0$ и квантовой ямы соответственно.

Для оценки величины $L$ в формулах (3), (5) для ямы, ширина $l$ которой обеспечивает существование квази-2D экситонов $\left(l_{\min }<l \lesssim a_{\mathrm{B}}\right)$, принимаем $\Phi(z)=(\sqrt{2 / \pi} / \tilde{a}) \psi^{2}(z)[7]$. Здесь $\psi(z)$ - нормированная на единицу функция размерного квантования в яме электрона и дырки, $\tilde{a}(l)-$ зависящий от $l$ вариационный радиус квази-2D экситона в интервале $1 / 2<\tilde{a} / a_{\mathrm{B}}<1$. При $l_{\min }<l \lesssim a_{\mathrm{B}}$ получаем $L=\sqrt{\pi / 2} a_{\mathrm{B}}^{3} / \tilde{a}^{2}$ в случае основного экситонного состояния, причем $l_{\min } \approx 5 \mathrm{HM}$ для ям $\mathrm{GaAs} / \mathrm{AlGaAs}[7,12]$ и $l_{\min } \approx 2$ нм для ям $\mathrm{ZnO} / \mathrm{ZnMgO}[13,14]$.

Из результатов работы [10] следует соотношение

$$
\frac{1}{\chi_{\alpha \alpha}^{0}}-\frac{1}{A^{3}}\left[S_{0}(0)+\mu S_{1}\left(\frac{2 h_{1}}{A}\right)\right] \approx \frac{3}{\eta a^{3}} \frac{u_{\alpha}^{2}-\omega^{2}-i \omega G_{\alpha}}{U_{\alpha}^{2}}
$$

для дипольных плазмонов, поляризованных по $\alpha$-оси наносфероида, находящегося в слое частиц вблизи поверхности полупроводника. В выражении (7) $u_{\alpha}^{2}=\omega_{p}^{2} \tilde{N}^{(\alpha)} / \tilde{\varepsilon}_{*}^{(\alpha)}, \quad G_{\alpha}=\gamma, \quad U_{\alpha}^{2}=\omega_{p}^{2} \varepsilon_{1} /\left(\tilde{\varepsilon}_{*}^{(\alpha)}\right)^{2}, \quad$ где $\tilde{N}^{(\alpha)}=N^{(\alpha)}-(\eta / 3)(a / A)^{3}\left[S_{0}(0)+\mu S_{1}\left(2 h_{1} / A\right)\right], \quad \tilde{\varepsilon}_{*}^{(\alpha)}=$ $=\left(\varepsilon_{\infty}-\varepsilon_{1}\right) \tilde{N}^{(\alpha)}+\varepsilon_{1}$. Подстановка (7) в (5) дает для эффективной поляризуемости плазмон-экситонного комплекса формулу

$\chi_{\alpha \alpha}(\omega)=\frac{\eta a^{3}}{3} \frac{U_{\alpha}^{2}\left(\omega_{\alpha}^{2}-\omega^{2}-2 i \omega \Gamma\right)}{\left(\omega_{\alpha}^{2}-\omega^{2}-2 i \omega \Gamma\right)\left(u_{\alpha}^{2}-\omega^{2}-i \omega G_{\alpha}\right)-W^{4}}$, где $\alpha=x, y$. В резонансный знаменатель выражения (8) входят комплексные частоты $\omega_{\alpha}-i \Gamma$,темных“ экситонов квантовой ямы в отсутствие наночастиц и $u_{\alpha}-i G_{\alpha} / 2$ плазмонов наночастиц в отсутствие квантовой ямы. С учетом соотношения $\omega_{\alpha} \approx \omega_{0}$, где $\omega_{0}-$ частота 3D-экситона в материале квантовой ямы, получаем

$$
W^{4}=\frac{\eta a^{3} L}{8 h^{4}}\left(1-\mu^{2}\right) \omega_{L T} \omega_{0} U_{\alpha}^{2} S_{W}\left(\frac{2 h}{A}\right) .
$$

Эта константа характеризует кулоновское взаимодействие плазмонных и экситонных возбуждений при $a, L, h, A \ll c / \omega$. В (9) $S_{W}(\xi) \equiv-\xi^{5} S_{2}(\xi)>0-$ функция, монотонно убывающая от значения $S_{W}(0)=1$, которое при $2 h / A \ll 1$ соответствует взаимодействию плазмона частицы только с собственным „изображением“6 в квантовой яме, до $S_{W}(\zeta) \ll 1$ при $\zeta>2$.

Применим теперь теоретические результаты к квази$2 \mathrm{D}$ экситонам $e_{1}-h h_{1}$ квантовых ям GaAs/AlGaAs [7-9], к экситонам А и В ям на основе $\mathrm{ZnO} / \mathrm{ZnMgO}[13,14]$ и в последнем случае сопоставим результаты теории с экспериментом [4]. Входящие в выражения (7)-(9) величины $u_{\alpha}, U_{\alpha}$ и $G_{\alpha}$ находим, используя параметры функции $\varepsilon(\omega)$ металла, которые получаем при аппроксимации данных спектроскопии вблизи частоты $\omega_{0}$ экситона соответствующей квантовой ямы. На основе данных работы [15] находим $\hbar \omega_{p}=9.3$ эВ, $\hbar \gamma=0.03$ эВ и $\varepsilon_{\infty}=4.1$ для серебра вблизи $\hbar \omega_{0}=1.5$ эВ, а на основе данных [16] получаем $\hbar \omega_{p}=13.6$ эВ, $\hbar \gamma=0.52$ эВ и $\varepsilon_{\infty}=1$ для алюминия вблизи $\hbar \omega_{0}=3.4$ эВ. Для экситонов в квантовых ямах $\mathrm{GaAs}$ и $\mathrm{ZnO}$ используются значения $\varepsilon_{b}, \omega_{L T}$ и $a_{\mathrm{B}}$, приведенные в таблице. Параметр нерадиационного затухания экситонов при их рассеянии на акустических и оптических (с частотой $\omega_{L O}$ ) фононах оцениваем по формуле $\Gamma(T)=A T+B\left[\exp \left(\hbar \omega_{L O} / k T\right)-1\right]^{-1}$. При $T<150 \mathrm{~K}$ для $e_{1}-h h_{1}$ экситонов в квантовых ямах GaAs co значениями $A$ и $B$ из работы [17] находим $\hbar \Gamma<0.4$ мэВ, а для экситонов в ямах $\mathrm{ZnO}$ с $A$ и $B$ из работы [13] получаем $\hbar \Gamma<6$ мэВ.

Результаты расчета спектра $\operatorname{Im} \chi_{\alpha \alpha}(\hbar \omega)$ поляризуемости (8) плазмон-экситонного комплекса представлены на рис. 2, $a$ для наноструктур GaAs/Ag и на рис. 3, $a$ - для структур $\mathrm{ZnO} / \mathrm{Al}$. Физически величина $\operatorname{Im} \chi_{\alpha \alpha}$ выражает спектр поглощения света плазмон-экситонными комплексами в структуре, показанной на рис. 1. При расчете спектра $\operatorname{Im} \chi_{\alpha \alpha}$ с параметрами из таблицы фиксируется 
частота экситона $\omega_{\alpha}=\omega_{0}$, а частота плазмона $u_{\alpha}$ отстраивается от $\omega_{0}$ путем изменения параметра $\eta$, определяющего форму (сплюснутость) металлических сфероидов. На рис. 2, $a$ и 3, $a$ показано, что при плазмон-экситонном резонансе $u_{\alpha}=\omega_{0}$ в наноструктурах $\mathrm{GaAs} / \mathrm{Ag}$ и $\mathrm{ZnO} / \mathrm{Al}$ спектры $\operatorname{Im} \chi_{\alpha \alpha}$ состоят из двух пиков, разделенных провалом при энергии экситона $\hbar \omega_{0}$. При отстройке частоты $u_{\alpha}$ от $\omega_{0}$ пики разделяются на плазмонный и
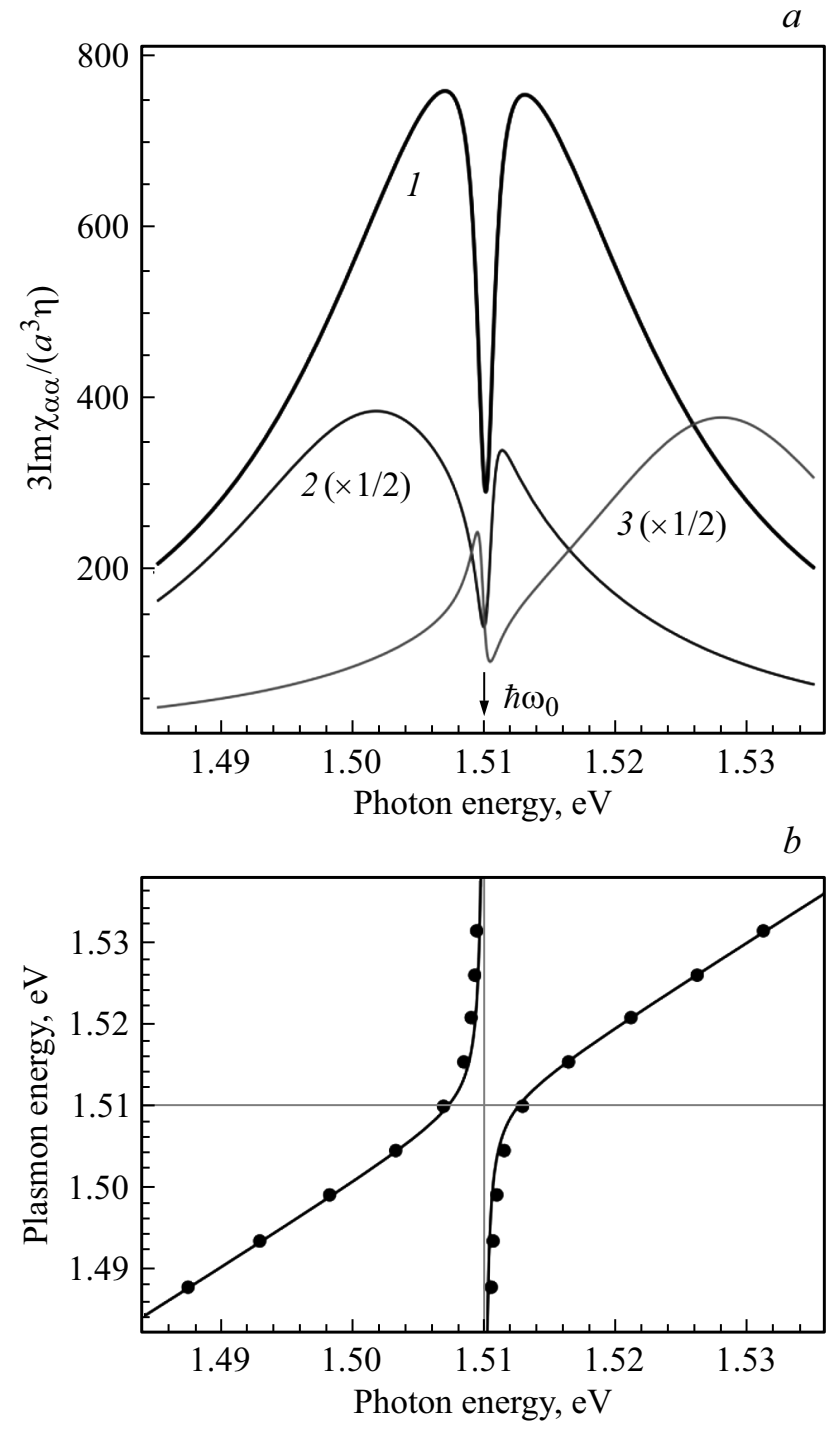

Рис. 2. $a-$ спектры поляризуемости (8) плазмон-экситонных комплексов в наноструктурах $\mathrm{GaAs} / \mathrm{Ag}$ при разных энергиях $\hbar u_{\alpha}$ плазмонов в частицах $\mathrm{Ag}$ вблизи резонанса $u_{\alpha}=\omega_{0}$ с экситоном $e_{1}-h h_{1}$ квантовой ямы GaAs/AlGaAs $\left(\hbar \omega_{0}=1.51\right.$ эB). Спектры соответствуют следующим значениям $\eta: 1-0.148$ $\left(u_{\alpha}=\omega_{0}\right), 2-0.145\left(u_{\alpha}<\omega_{0}\right), 3-0.153\left(u_{\alpha}>\omega_{0}\right) . b-$ энергии максимумов спектра $\operatorname{Im} \chi_{\alpha \alpha}$ (точки) и энергии $\hbar \omega_{ \pm}$ из (10) (сплошные линии) при отстройке $u_{\alpha}$ от $\omega_{0}$. Вычислено с параметрами экситонов и плазмонов из таблицы, $\varepsilon_{1}=2.25$ и следующими геометрическими параметрами, нм: $a=10$, $A=40, h_{1}=5, h=10, L=19$. Параметр радиационного затухания $\hbar \Gamma_{0, x}=40$ мкэВ при $\kappa=0$ соответствует $l \approx 10$ нм в соответствии с [9].
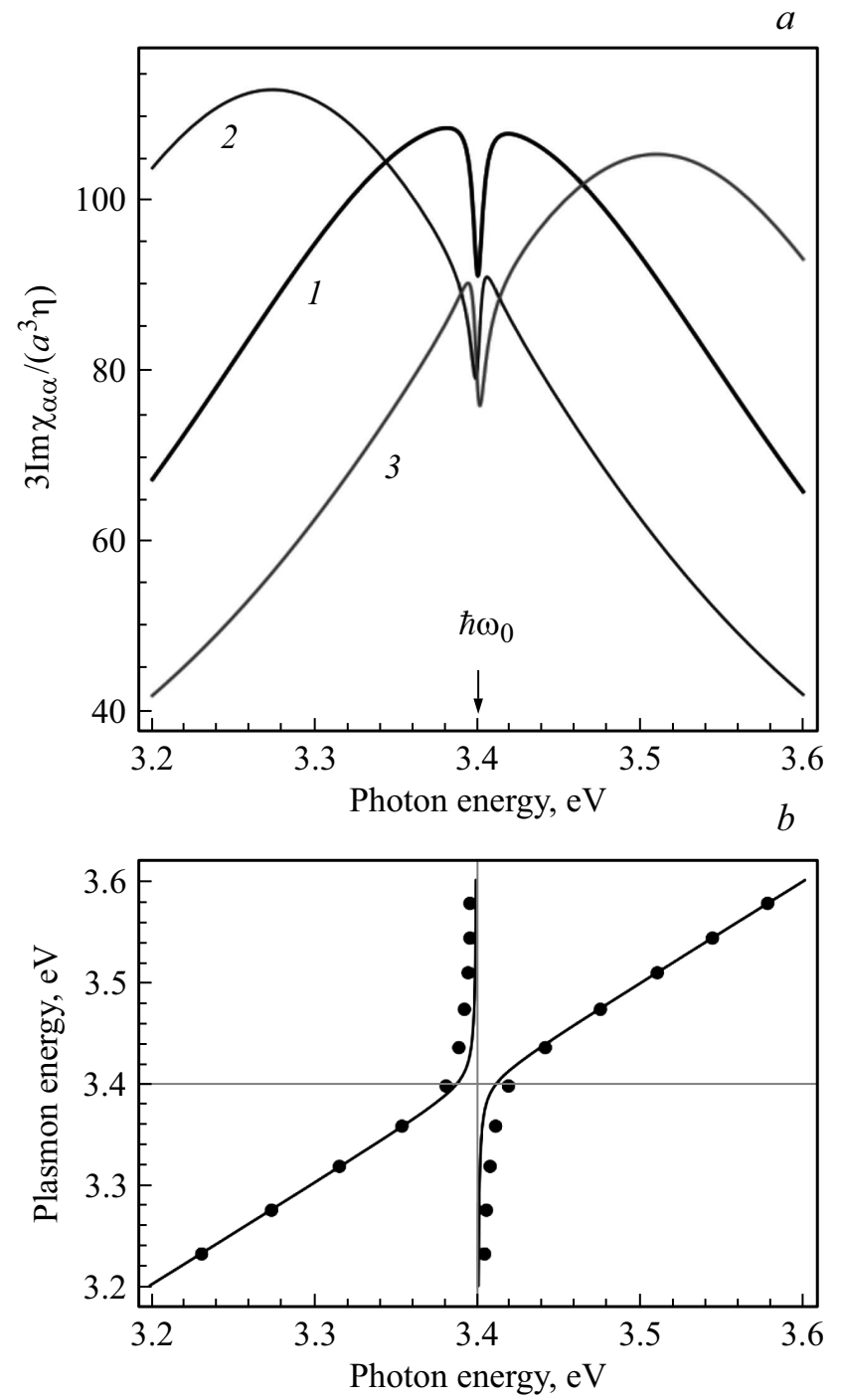

Pис. 3. $a-$ спектры поляризуемости (8) плазмон-экситонных комплексов в наноструктурах $\mathrm{ZnO} / \mathrm{Al}$ при разных энергиях $\hbar u_{\alpha}$ плазмонов в частицах $\mathrm{Al}$ вблизи резонанса $u_{\alpha}=\omega_{0}$ с экситоном квантовой ямы $\mathrm{ZnO} / \mathrm{MgZnO}\left(\hbar \omega_{0}=3.4\right.$ эВ). Спектры соответствуют следующим значениям $\eta: 1-0.158\left(u_{\alpha}=\omega_{0}\right)$, $2-0.143\left(u_{\alpha}<\omega_{0}\right), 3-0.173\left(u_{\alpha}>\omega_{0}\right) . b-$ энергии максимумов спектра $\operatorname{Im} \chi_{\alpha \alpha}$ (точки) и энергии $\hbar \omega_{ \pm}$из $(10)$ (сплошные линии) при отстройке $u_{\alpha}$ от $\omega_{0}$. Вычислено с параметрами экситонов и плазмонов из таблицы, $\varepsilon_{1}=1$ и следующими геометрическими параметрами, нм: $a=10, A=40, h_{1}=5$, $h=10, L=6.7$. При этом $l \approx 2.7$ нм соответствует параметру радиационного затухания $\hbar \Gamma_{0, x}=0.6$ мэВ при $\kappa=0$ [14].

экситонный, для которых частоты максимумов при некоторых значениях $u_{\alpha}$ показаны точками на рис. $2, b$ для структур $\mathrm{GaAs} / \mathrm{Ag}$ и на рис. $3, b$ для $\mathrm{ZnO} / \mathrm{Al}$. Видно, что зависимость от $u_{\alpha}$ частот, соответствующих максимумам рассчитанного спектра $\operatorname{Im} \chi_{\alpha \alpha}$, обладает признаками антипересечения, характерного для расщепления Раби.

Подчеркнем, что точки на рис. $2, b$ и $3, b$ относятся к случаю, когда расстояние между спектральными пиками при $u_{\alpha}=\omega_{0}$ существенно меньше ши- 
рины одного из резонансов (согласно таблице плазмонного с $G_{\alpha}=\gamma \gg 2 \Delta$, где $\left.\Delta=W^{2} /\left(2 \omega_{0}\right)\right)$. Для плазмон-экситонов эта ситуация довольно общая, так как обычно экситонные резонансы узкие $\left(\Gamma / \omega_{0} \sim 10^{-3}\right)$ и слабые $\left(\Gamma_{0, x} /\left(\Gamma+\Gamma_{0, x}\right) \lesssim 1\right)$, а плазмонные резонансы относительно широкие $\left(\gamma / 2 \Gamma \sim 10^{2}\right)$ и сильные $\left(U_{\alpha} / \gamma \sim 10-10^{2}\right)$. Наличие на рис. $2, a$ и $3, a$ двухпиковой структуры спектра при $2 \Delta \ll \gamma$ обусловлено узостью второго (экситонного) резонанса $(\Gamma \ll \Delta)$, а нарушение этого условия при $\gamma, 2 \Gamma \gtrsim 2 \Delta$ должно привести к подавлению двухпиковой структуры спектра.

Сравним поведение максимумов в спектрах $\operatorname{Im} \chi_{\alpha \alpha}(\omega)$, вычисленных из (8), при расстройке частот $u_{\alpha}$ и $\omega_{0}$, с антипересечением спектров, полученным в элементарной модели связанных плазмонного и экситонного осцилляторов без затухания. Для этого из резонансного знаменателя поляризуемости (8) при $\Gamma=\gamma=0$ получаем частоты

$$
\omega_{ \pm}=\frac{\omega_{0}+u_{\alpha}}{2} \pm \sqrt{\left(\frac{\omega_{0}-u_{\alpha}}{2}\right)^{2}+\Delta^{2}},
$$

определяющие расщепление $\omega_{+}-\omega_{-}=2 \Delta$ при $u_{\alpha}=\omega_{0}$. Их зависимость от частоты плазмона $u_{\alpha}$ при $\omega_{\alpha}=\omega_{0}$ показана на рис. 2, $b$ и $3, b$ непрерывными линиями, которые выражают расщепление Раби в форме антипересечения спектров. Существенно, что на эти зависимости хорошо ложатся точки, соответствующие положениям пиков в спектрах $\operatorname{Im} \chi_{\alpha \alpha}(\omega)$, рассчитанных из (8) при разных $u_{\alpha}$ с реальными параметрами затухания плазмонов и экситонов. Отметим также, что вычисленное выше расстояние $\hbar\left(\omega_{+}-\omega_{-}\right)=24$ мэВ между пиками при плазмон-экситонном резонансе близко к величине расщепления Раби 15 мэВ, измеренной в работе [4] для наноструктур $\mathrm{ZnO} / \mathrm{Al}$. Этот факт позволяет констатировать, что представленная выше теория находится в соответствии с данными эксперимента.

Итак, в статье представлены результаты электродинамической теории плазмон-экситонов. Для модели близко расположенных квантовой ямы и металлических наночастиц рассчитаны спектры поляризуемости плазмонэкситонных комплексов, которые определяют наблюдаемые оптические спектры. Показано, что положения пиков в спектре вблизи плазмон-экситонного резонанса имеют признаки расщепления Раби с найденной в данной работе константой взаимодействия возбуждений.

\section{Финансирование работы}

Работа выполнена при финансовой поддержке РФФИ (проект № 18-02-00668) и правительства РФ (договор 14.W03.31.0011, лаборатория Оптики кристаллов и гетероструктур с экстремальной двумерностью, рук. Б. Жиль - В. Gil).

\section{Конфликт интересов}

Авторы заявляют, что у них нет конфликта интересов.

\section{Список литературы}

[1] Y. Sugawara, T.A. Kelf, J.J. Baumberg, M.E. Abdelsalam, P.N. Bartlett. Phys. Rev. Lett., 97, 266808 (2006).

[2] S. Balci. Optics Lett., 38, 4498 (2013).

[3] P. Vasa, R. Pomraenke, S. Schwieger, Yu.I. Mazur, Vas. Kunets, P. Srinivasan, E. Johnson, J.E. Kihm, D.S. Kim, E. Runge, G. Salamo, C. Lienau. Phys. Rev. Lett., 101, 116801 (2008).

[4] B.J. Lawrie, K.-W. Kim, D.P. Norton, R.F. Haglund, jr. Nano Lett., 12, 6152 (2012).

[5] В.А. Кособукин. ФТТ, 57, 1413 (2015).

[6] V.A. Kosobukin. Solid State Commun., 228, 43 (2016).

[7] E.L. Ivchenko. Optical spectroscopy of semiconductor nanostructures (Alpha Science Internat., Ltd, 2005).

[8] E.L. Ivchenko, V.P. Kochereshko, P.S. Kop'ev, V.A. Kosobukin, I.N. Uraltsev, D.R. Yakovlev. Solid State Commun., 70, 529 (1989).

[9] E.S. Khramtsov, P.A. Belov, P.S. Grigoryev, I.V. Ignatiev, S.Yu. Verbin, Yu.P. Efimov, S.A. Eliseev, V.A. Lovtcius, V.V. Petrov, S.L. Yakovlev. J. Appl. Phys., 119, 184301 (2016).

[10] В.А. Кособукин, А.В. Коротченков. ФТТ, 58, 2446 (2016).

[11] V.L. Berkovits, V.A. Kosobukin, A.B. Gordeeva. J. Appl. Phys., 118, 245305 (2015).

[12] R.L. Greene, K.K. Bajaj, D.E. Phelps. Phys. Rev. B, 29, 1807 (1984).

[13] T. Makino, Y. Segawa, M. Kawasaki, H. Koinuma. Semicond. Sci. Technol., 20, S78 (2005).

[14] М.Н. Батаев, Н.Г. Философов, А.Ю. Серов, В.Ф. Агекян, C. Mohrain, В.П. Кочерешко. ФТТ, 60, 2450 (2018).

[15] P.B. Johnson, R.W. Christy. Phys. Rev. B, 6, 4370 (1972).

[16] K.M. McPeak, S.V. Jayanti, S.J.P. Kress, S. Meyer S. Iotti, A. Rossinelli, D.J. Norris. ACS Photonics, 2, 326 (2015).

[17] J. Lee, E.S. Koteles, M.O. Vassell. Phys. Rev. B, 33, 5512 (1986).

Редактор А.Н. Смирнов

\section{On the theory of plasmon-excitons: estimation of coupling constant and optical spectra}

\section{N.S. Averkiev, A.V. Korotchenkov, V.A. Kosobukin}

loffe Institute, 194021 St. Petersburg, Russia

\begin{abstract}
The structure of optical spectrum due to resonant coupling of quasi-two-dimensional excitons and local plasmons is studied theoretically. The constant of plasmon-exciton coupling is estimated in a model of closely located a quantum well in semiconductor and a layer of metal nanoparticles in adjacent dielectric. From numerical calculations for the nanostructures $\mathrm{GaAs} / \mathrm{Ag}$ and $\mathrm{ZnO} / \mathrm{Al}$ it is concluded that the polarizability spectrum of plasmon-exciton complexes has two-peak structure at the resonance between excitations of the two types. Behavior of the spectral structure in detuning the resonance demonstrates the anticrossing of plasmon and exciton spectra which is a sign of the plasmon-excitonic Rabi splitting.
\end{abstract}

